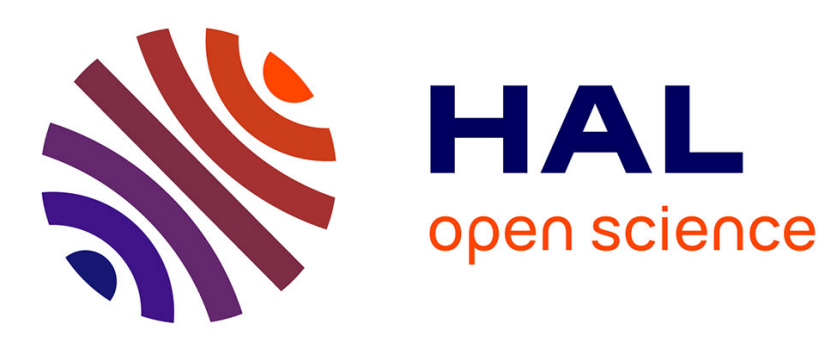

\title{
Responsabilité individuelle et fiscalité
}

Louis Lévy-Garboua, Claude Montmarquette, Marie Claire Villeval

\section{To cite this version:}

Louis Lévy-Garboua, Claude Montmarquette, Marie Claire Villeval. Responsabilité individuelle et fiscalité. Economie et Prévision, 2008, 182 (1), pp.19-31. halshs-00276094

\section{HAL Id: halshs-00276094 \\ https://shs.hal.science/halshs-00276094}

Submitted on 28 Apr 2008

HAL is a multi-disciplinary open access archive for the deposit and dissemination of scientific research documents, whether they are published or not. The documents may come from teaching and research institutions in France or abroad, or from public or private research centers.
L'archive ouverte pluridisciplinaire HAL, est destinée au dépôt et à la diffusion de documents scientifiques de niveau recherche, publiés ou non, émanant des établissements d'enseignement et de recherche français ou étrangers, des laboratoires publics ou privés. 


\section{Responsabilité individuelle et fiscalité}

Louis Lévy-Garboua $^{\mathrm{a}}$, Claude Montmarquette $^{\mathrm{b}}$, Marie-Claire Villeval $^{\mathrm{c}}$

Nous remercions Mark Isaac, nos arbitres et les participants de plusieurs colloques et séminaires pour leurs commentaires sur une version antérieure de ce texte. Nous remercions également R. Zeiliger pour la programmation de l'expérience et N. Viennot. La recherche a bénéficié d'un soutien financier du ministère de la recherche français (ACI International) et du Gouvernement du Québec.

a TEAM-CES, Université de Paris I, 106-112 Bd de l'Hôpital 75647 Paris Cedex 13, France, et CIRANO, Montréal, Canada.

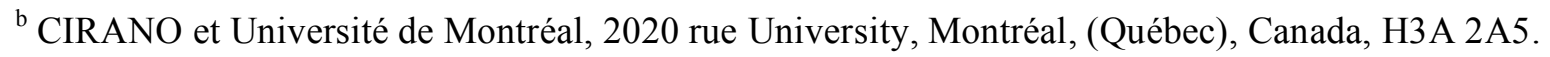

${ }^{c}$ CNRS - GATE, 93, chemin des Mouilles 69130 Ecully, France et Institute for the Study of Labor (IZA), Bonn, Allemagne.villeval@gate.cnrs.fr 


\section{Résumé long}

Lorsque le financement d'un bien collectif repose sur des contributions volontaires, il est courant d'observer une offre insuffisante du bien collectif en raison d'un comportement de passager clandestin. Pour faire face à un déficit d'offre de bien collectif, la puissance publique intervient généralement pour garantir la fourniture du service à tous, en contrepartie d'une prise en charge publique de son coût. La fiscalité mise en place doit assurer l'accès de tous au service. Tout concepteur de politique est alors à la recherche d'un principe qui permette de respecter justice et efficacité. Une application majeure de cette réflexion est le domaine de l'assurance-maladie. En cas de déficit du système de protection sociale, est-il plus efficace de répartir égalitairement la charge fiscale impliquée par le traitement du déficit ou bien de moduler cette charge en fonction de critères individualisés (par exemple le degré d'usage du service comme avec le ticket modérateur, ou bien l'effort de contribution volontaire à sa prise en charge)? Il s'agit d'identifier un régime fiscal qui favorise la responsabilité individuelle.

L'objectif de cet article est d'évaluer l'efficacité comparée de deux régimes fiscaux dans un contexte de contribution volontaire au financement de biens collectifs. À l'aide d'une expérience de laboratoire réalisée au Québec et en France, nous étudions le comportement individuel de contribution à un fonds commun destiné à compenser les victimes d'un dommage entraînant la perte du revenu courant. Au moment où les individus contribuent, ils connaissent la probabilité individuelle d'être victime du dommage, mais ils ne savent pas encore qui seront les victimes. En cas d'insuffisance du fonds commun, deux types de gestion du déficit sont envisagés. Dans un premier traitement, un impôt uniforme (i.e. une "flat tax" égale pour chaque membre du groupe quelle que soit sa contribution volontaire) est prélevé a posteriori pour combler le déficit, moyennant un surcoût fiscal. Dans un second traitement, le montant de l'impôt supporté par chaque individu en cas de déficit est modulé en fonction de sa contribution volontaire.

L'optimum social est intérieur et identique dans ces deux traitements : il correspond à une répartition égalitaire entre les membres du groupe de la totalité des pertes subies par les victimes. En revanche, l'équilibre de Nash est totalement distinct. Lorsque l'impôt est uniforme, l'équilibre est atteint lorsque personne ne contribue malgré le surcoût du prélèvement. Lorsque l'impôt est individualisé, un équilibre symétrique correspond à l'optimum : assurer par sa contribution la prise en charge de la totalité des pertes subies par les victimes assure la compensation des pertes et évite le prélèvement fiscal et son coût additionnel. Il existe d'autres équilibres non symétriques mais déficitaires. La comparaison des contributions dans ces deux traitements permet d'identifier quelle institution favorise le plus la responsabilisation des individus et leur coopération pour le financement du bien.

Les résultats expérimentaux confortent notre modèle théorique. L'impôt uniforme assure l'ensemble des victimes contre la perte de leur revenu en cas de dommage, mais il suscite un effet d'éviction sur les contributions volontaires et génère un coût supplémentaire pour la collectivité (i.e. le coût additionnel de prélèvement) qui grève l'efficience. Au contraire, l'impôt individualisé conduit à une contribution moyenne proche à la fois de l'équilibre symétrique et de l'optimum, et favorise de manière stable la coopération entre participants pour contribuer au fonds commun. Sans parvenir à supprimer le comportement de passager clandestin, ce mécanisme responsabilise les individus au financement du bien et les prémunit contre le risque d'exploitation par les resquilleurs. Nous montrons donc que l'efficacité est améliorée par une responsabilisation des individus au coût réel du service. 


\section{Introduction}

Lorsque le financement d'un bien collectif repose sur des contributions volontaires, il est rationnel pour les individus d'adopter un comportement de passager clandestin. Dès lors, il est courant d'observer une offre insuffisante du bien collectif. Pour faire face à un déficit d'offre de bien collectif, la puissance publique intervient généralement pour garantir la fourniture du service à tous, en contrepartie d'une prise en charge publique de son coût. La fiscalité mise en place doit donc assurer l'accès de tous au service ou au bien. Tout concepteur de politique est alors à la recherche d'un principe qui permette de respecter à la fois la justice et l'efficacité. Une application majeure de cette réflexion est le domaine de la santé et de l'assurancemaladie. En cas de déficit du système de protection sociale, est-il plus efficace d'adopter une répartition égalitaire de la charge fiscale impliquée par le traitement du déficit ou bien de moduler cette charge en fonction de critères individualisés (par exemple le degré d'usage du service comme avec le ticket modérateur, ou bien l'effort de contribution volontaire à sa prise en charge)? L'interrogation porte alors sur l'identification d'un régime fiscal qui favorise la responsabilité individuelle.

L'objectif de cet article est d'évaluer l'efficacité comparée de deux régimes fiscaux dans un contexte de contribution volontaire au financement de biens collectifs. A l'aide d'une expérience de laboratoire réalisée simultanément au Québec et en France, nous étudions le comportement individuel de contribution à un fonds commun destiné à compenser les victimes d'un dommage entraînant la perte du revenu courant. Au moment où les individus contribuent, ils connaissent la probabilité individuelle d'être victime du dommage mais ils ne savent pas encore qui seront les victimes au sein de leur groupe. En cas d'insuffisance du fonds commun, deux types de gestion du déficit sont envisagés. Dans un premier traitement, un impôt uniforme (i.e. une «flat tax», égale pour chaque membre du groupe quelle que soit sa contribution volontaire) est prélevé a posteriori pour combler le déficit, moyennant un 
surcoût fiscal. Dans un second traitement, le montant de l'impôt supporté par chaque individu en cas de déficit est déterminé en fonction de sa contribution volontaire (i.e. l'impôt est réduit pour le sujet qui a contribué davantage à la première étape du jeu). L'optimum social est intérieur et identique dans ces deux traitements : il correspond à une répartition égalitaire entre tous les membres du groupe de la totalité des pertes subies par les victimes du dommage. En revanche, l'équilibre de Nash est totalement distinct dans ces deux traitements. Lorsque l'impôt est uniforme, l'équilibre est atteint lorsque personne ne contribue malgré le surcoût du prélèvement. Lorsque l'impôt est individualisé, un équilibre symétrique correspond à l'optimum : assurer par sa contribution la prise en charge de la totalité des pertes subies par les victimes permet non seulement d'assurer la compensation des pertes mais aussi d'éviter le prélèvement fiscal et son coût additionnel. Il existe d'autres équilibres non symétriques qui sont alors déficitaires. Les données expérimentales permettent de déterminer quel est l'équilibre sélectionné par les joueurs. La comparaison des contributions dans ces deux traitements permet d'identifier quelle institution favorise le plus la responsabilisation des individus et leur coopération pour le financement du bien collectif. L'hypothèse que nous testons dans cet article est que l'efficacité est améliorée par une responsabilisation des individus au coût réel du service.

L'originalité de notre démarche par rapport aux nombreuses expériences utilisant le cadre du financement volontaire des biens publics (Cf. Davis et Holt, 1993; Ledyard,1995) peut se lire à plusieurs niveaux. Tout d'abord, nous nous intéressons à un bien collectif qui se distingue du bien public au sens où seules les victimes du dommage vont bénéficier du bien alors que le bien public pur ne peut exclure de bénéficiaire. Alors que les membres du groupe bénéficient habituellement du même rendement marginal du bien public, dans notre expérience, le rendement marginal du bien collectif peut être nul si l'on n'est pas désigné comme victime du dommage. Chez Goeree, Holt et Laury (2000), il y a aussi une différence entre le rendement 
interne et le rendement externe du bien public mais, dans notre expérience, l'individu ne sait pas au moment où il contribue s'il sera victime ou non. Une deuxième originalité de notre article peut également se lire par rapport à l'abondante littérature expérimentale sur la fiscalité (voir en particulier, Alm, 1991 et Torgler, 2004); elle provient de la possibilité de comparer l'efficacité d'un mécanisme uniforme et d'un mécanisme individualisé de taxation. L'individualisation est ici liée à la différenciation des comportements des individus et non pas à une différenciation de leur situation a priori (par différenciation des dotations, Isaac et Walker,1988, ou du rendement marginal individuel du bien public, Chan, Mestelman, Moir et Muller, 1999). Ce faisant, notre protocole permet de mesurer d'éventuels effets d'éviction («crowding-out ») (Bergstrom, Blume et Varian,1986 ; Roberts, 1987 ; Andreoni, 1993) des contributions volontaires par la fiscalité et leur sensibilité à la nature du régime fiscal. Une dernière originalité de notre approche est de permettre la comparaison entre deux jeux partageant le même optimum intérieur mais où l'équilibre de Nash diverge, l'un étant en coin et l'autre étant intérieur à l'espace de décision. Ceci permet non seulement de réduire les biais dus aux erreurs de décision (Keser, 1996 ; Holt et Laury, 2000), mais aussi de varier le risque de la coopération selon les traitements ${ }^{1}$.

Les résultats expérimentaux confortent le modèle théorique proposé. L’impôt uniforme permet d'assurer l'ensemble des victimes contre la perte de leur revenu en cas de dommage, mais il suscite un effet d'éviction sur les contributions volontaires et génère un coût supplémentaire pour la collectivité (correspondant au coût additionnel de prélèvement) qui grève l'efficience. Au contraire, l'impôt individualisé conduit à une contribution moyenne proche à la fois de l'équilibre symétrique et de l'optimum, et favorise la coopération entre participants pour contribuer au fonds commun et sa stabilité dans le temps. Sans parvenir à supprimer le comportement de passager clandestin, ce mécanisme responsabilise les individus au financement du bien et les prémunit contre le risque d'exploitation par les resquilleurs. 
Le reste de l'article est organisé comme suit. La section 2 présente le modèle théorique. La section 3 est consacrée au protocole expérimental. La section 4 analyse les principaux résultats statistiques et économétriques. Enfin, la section 5 conclut et discute des implications de nos observations.

\section{Le modèle}

Considérons un jeu de bien collectif en deux étapes. Dans la première étape, les individus reçoivent une dotation et contribuent volontairement à un fonds commun destiné à compenser les pertes subies par plusieurs membres du groupe frappés aléatoirement en début de seconde étape. Dans cette seconde étape, si le fonds commun s'avère insuffisant pour combler les pertes subies, un impôt est prélevé pour indemniser les victimes. Dans le cadre d'un traitement uniforme du déficit, chaque membre du groupe, quelle que soit sa contribution initiale, subit un surcoût lié au prélèvement obligatoire. Dans le cadre d'un traitement individualisé du déficit, l'impôt et son coût associé sont supérieurs si l'individu a moins contribué que les autres. Notre approche vise dans ces contextes à comprendre les déterminants de la coopération à souscrire volontairement au fonds commun, les équilibres réalisés et les stratégies retenues.

Deux cas peuvent se présenter :

1. Il est supérieur ou égal au total de la somme perdue par les membres du groupe. Tous recouvrent leurs pertes et l'excédent éventuel est brûlé.

2. Le fonds commun est insuffisant pour permettre aux victimes de retrouver leur dotation. Si le fonds commun est insuffisant, on fait face à un déficit budgétaire ex ante qu'il faudra financer ex post. Deux scénarios institutionnels de financement du déficit budgétaire sont ici considérés du point de vue du comportement des agents dans la phase de contribution volontaire : l'impôt uniforme et l'impôt individualisé. 
Pour chaque scénario, nous allons identifier un optimum social et un équilibre de Nash. L'optimum social est une manière de résumer l'objectif recherché par le gouvernement. Il est décrit par la préférence sociale d'un planificateur bienveillant et impartial qui considère seulement le gain net de chacun des agents pour planifier l'ensemble de leurs contributions individuelles. L'équilibre de Nash est une solution du jeu où chacun des joueurs adopte une stratégie qui consiste à maximiser son gain net étant donné la décision des autres. Si optimum et équilibre coïncident, le gouvernement a toutes les chances d'atteindre son objectif. Mais s'ils divergent, on peut redouter que ce ne soit pas le cas. C'est précisément ce que l'expérience cherche à confirmer.

Le modèle théorique suppose que chaque agent décide à chaque période d'investir ou non une partie de son revenu $Y$ dans un fonds commun qui sert à rembourser les membres touchés aléatoirement par un sinistre.

Soit $Y \equiv 100:$ le revenu (constant) de l'agent $i$ à chaque période,

$g_{i}$ : la contribution de l'agent $i$ au fonds commun $\left(g_{i} \geq 0\right)$,

$\tilde{d}_{k}$ : la perte subie par l'agent $k$, qui prend seulement deux valeurs : $d$ ou 0 . Cette perte est une variable aléatoire i.i.d. qui prend l'une ou l'autre de ces deux valeurs à chaque période.

Soit $\pi_{i}$, le profit ou gain net de $i$ à chaque période,

$S$ : le nombre d'agents sinistrés sur $N$ participants. Nous allons supposer que $S$ individus sur un total de $N$ subissent un sinistre et un seul à chaque période $(1 \leq S<N)$. Cette hypothèse simplifie la représentation du problème en faisant du nombre de sinistres $S$ et du montant total des sinistres $L\left(\sum_{k=1}^{S} \tilde{d}_{j} \equiv S d=L\right)$ des grandeurs certaines, rapprochant ainsi la situation d'un petit groupe expérimental de ce que la loi des grands nombres réaliserait naturellement dans un grand groupe. 
Soit $p \equiv \frac{S}{N}$ la probabilité (indépendante de la période) qu'un des participants dans un groupe de $\mathrm{N}$ soit sinistré à la suite de $S$ tirages sans remise. Dans notre expérience, $N=12, S=4$; donc, $p=1 / 3$.

\subsection{Traitement du déficit avec impôt uniforme ${ }^{2}$}

Soit

$\pi_{i}=\left\{\begin{array}{lll}100-g_{i}-\frac{1}{N}\left[L-\sum_{j=1}^{N} g_{j}\right]-\left[\beta+\frac{\alpha}{2 N}\left(L-\sum_{j=1}^{N} g_{j}\right)^{2}\right] & \text { si } & L>\sum_{j=1}^{N} g_{j} \\ 100-g_{i} & \text { si } & L \leq \sum_{j=1}^{N} g_{j}\end{array}\right.$

où $\alpha$ et $\beta>0$.

La première ligne de l'équation (1) définit le gain de l'agent $i$ sous la condition que le fonds commun est déficitaire (la somme des sinistres est supérieure à la somme des contributions individuelles au fonds commun). Après le revenu moins la contribution au fonds commun de $i$, le troisième terme de l'équation est la contribution de $i$ en impôt requis pour combler le déficit. Cette contribution est égale pour tous. Précisément, l'impôt de chacun est $1 / N$ fois le montant du déficit. Le terme entre les accolades est le coût administratif et social du déficit budgétaire comblé par un impôt. Nous utilisons une fonction de coût quadratique pour compenser le déficit éventuel afin de rendre compte de l'idée que plus un déficit dans la gestion des systèmes sociaux est important et plus l'ajustement est coûteux pour une société, par exemple en termes d'ampleur des réformes requises.

La deuxième ligne de l'équation est le gain de $i$ sous la condition que le fonds commun n'est pas déficitaire. Dans ce dernier cas, le gain est le revenu moins la contribution au fonds de $i$.

Puisque seul entre dans l'expression du gain le total des dommages au sein du groupe, ce total est certain. Par conséquent, la couverture complète des victimes supprime le risque. Le 
planificateur maximise simplement la somme des gains individuels en considérant, comme les gains ont la même expression pour tous, qu'ils doivent tous avoir la même contribution :

$$
g_{j} \equiv g_{i} \quad \forall j \neq i \in(1, \ldots, N)
$$

La fonction de gain par tête (1) se réécrit ainsi :

$$
\pi_{i}^{*}=\left\{\begin{array}{cl}
100-\frac{L}{N}-\left\{\beta+\frac{\alpha}{2 N}\left[L-N g_{i}\right]^{2}\right\} & \text { si } g_{i}<\frac{L}{N} \\
100-g_{i} & \text { si } g_{i} \geq \frac{L}{N}
\end{array}\right.
$$

La fonction $\pi_{i}^{*}$ a son maximum en $g_{i}=\frac{L}{N}$, et le budget est alors en équilibre ex ante.

L'équilibre de Nash est atteint quand chaque joueur $i$ maximise son profit étant donné les contributions des autres. Si $\bar{g}$ est la contribution moyenne des autres joueurs, le profit de $i$ est :

$\pi_{i}=\left\{\begin{array}{c}100-g_{i}-\frac{1}{N}\left[L-(N-1) \bar{g}-g_{i}\right]-\left[\beta+\frac{\alpha}{2 N}\left(L-(N-1) \bar{g}-g_{i}\right)^{2}\right] \text { si } g_{i}+(N-1) \bar{g}<L \\ 100-g_{i} \text { si } g_{i}+(N-1) \bar{g} \geq L\end{array}\right.$

S'il existe un équilibre de Nash sans déficit budgétaire, celui-ci ne peut être que la contribution égale pour tous qui permettrait d'atteindre l'équilibre budgétaire ex ante: $g_{i}=\frac{L}{N}, \forall i=(1, \ldots, N)^{3}$. Dans la situation de déficit budgétaire, le profit est donné par la première ligne de l'équation (3). Son maximum est obtenu pour une contribution nulle si la condition suivante est respectée : $\alpha L \leq N-1$, ce qui est le cas pour des valeurs raisonnables de $\alpha$ et, en particulier, dans notre expérience. Cette condition s'appliquant à tous les joueurs, tous ont alors une contribution nulle à l'équilibre. Des deux solutions potentielles qui ont été identifiées, seule la seconde est un équilibre de Nash si le surcoût administratif d'un déficit budgétaire n'est pas trop élevé. Un agent qui croit que personne ne contribue au fonds commun n'a évidemment jamais intérêt à permettre l'équilibre budgétaire par sa seule 
contribution. Par contre, un agent qui croit que tous les autres contribuent juste ce qu'il faut pour atteindre l'équilibre budgétaire a lui-même intérêt à contribuer moins qu'eux et à provoquer ainsi un déficit budgétaire (voir les détails de la démonstration en annexe).

\subsection{Traitement du déficit avec impôt individualisé}

Désormais, en cas de déficit budgétaire, celui-ci est comblé par un impôt prélevé en fonction de la contribution de chacun au fonds commun. Le gain de $i$ se définit de la façon suivante :

$$
\pi_{i}=\left\{\begin{array}{cr}
100-g_{i}-\frac{\frac{L}{N}-g_{i}}{L-\sum_{j=1}^{N} g_{j}}\left[L-\sum_{J=1}^{N} g_{j}\right]-\left\{\beta+\frac{\alpha}{2 N}\left[L-\sum_{j=1}^{N} g_{j}\right]^{2}\right\} & \text { si } \quad L>\sum_{j=1}^{N} g_{j} \\
100-g_{i} & \text { si } \quad L \leq \sum_{j=1}^{N} g_{j}
\end{array}\right.
$$

où le terme entre les accolades avec $\alpha, \beta>0$ est le coût administratif et social du déficit budgétaire. Le troisième terme rend compte de la contribution individualisée au déficit selon la contribution volontaire de chacun au fonds commun.

Après simplification,

$$
\pi_{i}=\left\{\begin{array}{lll}
100-\frac{L}{N}-\beta-\frac{\alpha}{2 N}\left[L-\sum_{j=1}^{N} g_{j}\right]^{2} & \text { si } & L>\sum_{j=1}^{N} g_{j} \\
100-g_{i} & \text { si } & L \leq \sum_{j=1}^{N} g_{j}
\end{array}\right.
$$

Puisque seul entre dans l'expression du profit le total des dommages, que nous avons supposé certain, ce total est certain. Par conséquent, comme dans le cas précédent, la couverture complète des victimes du dommage supprime le risque et la fonction de gain par tête conserve la même expression que dans l'équation (2).

Le maximum du profit est évidemment obtenu lorsque les sujets contribuent tous également $\frac{L}{N}$ pour atteindre l'équilibre budgétaire ex ante, qui définit encore une fois l'optimum social. 
Bien que l'optimum social ne dépende pas du caractère uniforme ou individualisé de l'impôt, l'individualisation a pour conséquence de faire aussi de l'optimum social un équilibre de Nash. En effet, si l'agent $i$ recherche le profit maximum étant donné la contribution moyenne des autres, le profit de $i$ a pour expression:

$$
\pi_{i}=\left\{\begin{array}{c}
100-\frac{L}{N}-\beta-\frac{\alpha}{2 N}\left(L-(N-1) \bar{g}-g_{i}\right)^{2} \text { si } g_{i}+(N-1) \bar{g}<L \\
100-g_{i} \text { si } g_{i}+(N-1) \bar{g} \geq L
\end{array}\right.
$$

Il existe un équilibre de Nash symétrique où tous les joueurs choisissent la même contribution et le budget est en équilibre ex ante. Intuitivement, l'individu sachant qu'il devra nécessairement payer sa part d'indemnisation préfèrera le faire ex ante plutôt qu'ex post pour éviter d'avoir à s'acquitter d'un surcoût administratif. Mais il existe aussi d'autres équilibres non symétriques, qui sont alors déficitaires. En effet, s'il y avait un équilibre non symétrique non déficitaire, aucun joueur rationnel ne voudrait lui-même contribuer, ce qui contredirait l'hypothèse de non-déficit. Dans les équilibres non symétriques, le déficit combiné au mécanisme d'imposition de chacun selon sa responsabilité individuelle de ce déficit garantit aux joueurs que leur générosité ne sera pas pénalisée. Tous les joueurs sont rendus solidaires par le fait qu'ils sont tous assurés du même gain ex post. En outre, comme ce gain est uniquement fonction du déficit budgétaire ex ante et augmente rapidement avec lui (à cause du terme quadratique), certains joueurs peuvent contribuer au-delà de l'optimum social dans le but de limiter ce déficit et le surcoût administratif qu'il induit.

\section{Protocole expérimental}

Notre expérience est une variante d'un jeu de contribution volontaire au financement d'un bien public. Douze participants forment un groupe $(N=12)$. Chaque participant reçoit une dotation initiale de 100 jetons. Chacun peut alimenter le fonds commun en choisissant une 
contribution individuelle entre 0 et 100 jetons $\left(g_{i} \in[0,100]\right)$. Quatre des douze membres du groupe sont ensuite aléatoirement affectés par le dommage $(S=4)$. La perte totale est donc de 400 jetons $(L=400)$. La répartition égalitaire de la couverture complète de la somme des dommages correspond donc à une contribution individuelle de $\frac{L}{N}=33,33$ (approchée dans l'expérience par 33 ou 34). Les paramètres du surcoût fiscal sont $\alpha=0.005$ et $\beta=2$. En outre, avant la première période, chaque sujet a reçu un capital de départ destiné à couvrir d'éventuelles pertes temporaires. Chaque session consiste en 50 périodes afin de pouvoir observer la dynamique longue de contribution.

Six sessions expérimentales ont été réalisées pour chaque traitement, impliquant douze participants chacune. Afin de saisir un éventuel effet culturel, notre expérience a été organisée pour chaque traitement, pour moitié au Québec (LUB-3CE au CIRANO, Montréal) et pour moitié en France (laboratoire GATE, Lyon) aux mêmes dates. L'expérience a mobilisé 144 étudiants en provenance de diverses écoles d'ingénieurs et d'écoles de commerce. L’expérience a été réalisée à l'aide du logiciel REGATE (Zeiliger, 2000).

Une fois tous les participants présents, chacun a tiré au sort dans une enveloppe une étiquette indiquant son numéro d'ordinateur. Après lecture à voix haute des instructions (disponibles sur demande auprès des auteurs), les questions des participants ont reçu une réponse en privé et ces participants ont dû répondre à une série de questions visant à vérifier leur compréhension des instructions. Ces dernières ont été rédigées en termes neutres. La durée moyenne des sessions a été d'une heure trente. Le paiement a été effectué dans une salle séparée afin de préserver la confidentialité des gains. Le taux de conversion adopté est de 300 jetons $=1.55$ Dollar Canadien $=1$ Euro. Les gains moyens des participants à l'expérience ont été de 35 Dollars Canadiens ou 23 Euros. 
Les participants ont eu la possibilité d'opter en début de session pour la participation ou non à un tirage au sort réalisé en fin de session au moment du paiement des gains. Les participants avaient le choix entre recevoir une indemnité de participation fixe de 5 Dollars (2 Euros) venant s'ajouter aux gains réalisés lors de la session ou participer en fin de session à une loterie dans laquelle ils avaient $50 \%$ de chance de gagner 11 Dollars (5 Euros) supplémentaires ou bien 0. Ceci donne une indication (certes sommaire) sur l'aversion au risque des participants : ceux qui choisissent la loterie sont considérés moins riscophobes que les autres. Au moment du paiement, les participants ayant opté pour le tirage au sort procédaient eux-mêmes au lancement d'une pièce déterminant le montant de leur gain supplémentaire. De cette manière, la mesure de l'aversion au risque demeure exogène par rapport aux choix effectués dans le jeu de contribution.

\section{Résultats}

Nous présentons tout d'abord l'analyse générale des niveaux de contribution avant d'étudier les déterminants des comportements individuels puis l'hétérogénéité de ces comportements.

\subsection{Uniformité, individualisation et niveaux de contribution}

Les contributions individuelles au fonds commun sont en moyenne de 10,5 (écart-type : 15,3) dans le traitement avec impôt uniforme et de 27,2 (écart-type : 22,6) dans le traitement avec impôt individualisé. Le pourcentage de contributions nulles est respectivement de $38 \%$ et 14\%. La Figure 1 présente l'évolution des contributions moyennes au cours des 50 périodes pour chacun des deux traitements. Elle appelle aussitôt plusieurs observations. Tout d'abord, on peut remarquer une forte proximité des comportements en première période, ce qui suggère qu'en l'absence d'information sur le comportement des autres les participants suivent une norme a priori. En revanche, l'évolution des comportements devient très différente lorsque les 
individus sont informés du comportement des autres. Alors que la contribution moyenne se stabilise dans le traitement avec impôt individualisé, le resquillage (free-riding) se développe très rapidement et régulièrement au cours du temps quand l'impôt est uniforme ${ }^{4}$. Il est donc remarquable de constater que bien que notre jeu introduise des changements par rapport au jeu standard de bien public, nous observons néanmoins des comportements semblables de resquillage. Il est aussi important d'observer que si l'impôt est individualisé, les contributions se rapprochent de l'optimum social mais l'atteignent rarement, même si équilibre et optimum peuvent coïncider. Ceci est à rapprocher des résultats observés dans le jeu de Saijo et Nakamura (1995) qui utilise la même coïncidence. Ces derniers expliquent ce comportement par une préoccupation malveillante des joueurs dont la priorité est non pas le montant total des gains mais le classement entre les gains des individus. Si la fréquence relative des contributions situées à l'optimum reste faible, même dans le traitement avec impôt individualisé, elle augmente toutefois avec le temps dans ce traitement (de 8\% lors de la première période à $12 \%$ au cours des cinq dernières périodes), alors qu'elle diminue très rapidement dans le traitement avec impôt uniforme (de 12\% lors de la première période à $2 \%$ au cours des cinq dernières périodes). 


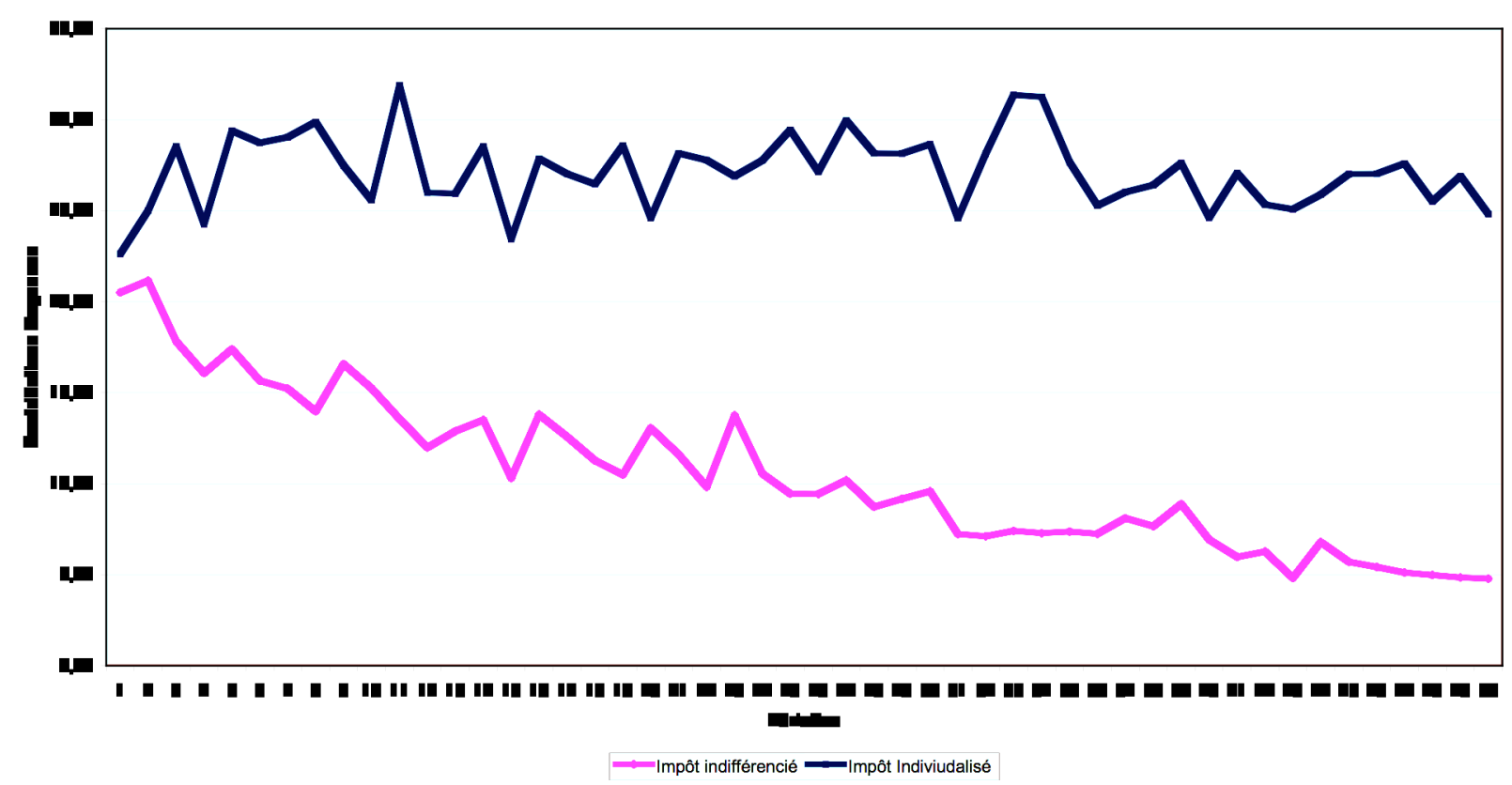

Figure 1. Évolution des contributions moyennes au cours du temps par traitement

L'analyse économétrique de l'évolution des contributions moyennes par traitement (Tableau 1) confirme que la contribution des participants au fonds commun diminue au cours du temps lorsque l'impôt est uniforme, mais pas dans le traitement avec impôt individualisé. Sur l'ensemble des 50 périodes, la contribution moyenne dans ce dernier traitement n'est inférieure que de 6 unités à l'optimum social. Si l'on estime la convergence asymptotique en faisant tendre $t$ vers l'infini dans le traitement où l'équilibre de Nash correspond à une contribution nulle, il apparaît que les joueurs devraient atteindre l'équilibre à la $63^{\text {ème }}$ période.

Tableau 1. Effet du temps sur la contribution moyenne par traitement (MCO)

\begin{tabular}{lcccc}
\hline & \multicolumn{2}{c}{ Impôt Uniforme } & \multicolumn{2}{c}{ Impôt Individualisé } \\
\hline & coeff. & t-stat & coeff. & t-stat \\
\hline Période & -0.276 & -20.45 & -0.014 & -0.70 \\
Constante & 17.543 & 44.37 & 27.520 & 47.40 \\
\hline Nombre d'observations & \multicolumn{2}{c}{50} & \multicolumn{2}{c}{50} \\
$\bar{R}^{2}$ corrigé & \multicolumn{2}{c}{0.895} & \multicolumn{2}{c}{-0.010} \\
\hline
\end{tabular}


La Figure 2 permet d'apprécier la dispersion des contributions individuelles et son évolution au cours de plusieurs ensembles de périodes. La médiane (que figure la droite au sein des barres) chute rapidement dans le traitement uniforme, alors que le traitement par l'impôt individualisé connaît une hausse de la médiane jusqu'à la dernière catégorie de périodes. Par ailleurs, si l'écart entre le premier et le troisième quartile (base et sommet des barres) se réduit au cours du temps quand l'impôt est uniforme, ce n'est pas le cas lorsque l'impôt est individualisé. Ceci laisse à penser que, même si les contributions se rapprochent en moyenne de l'optimum, une certaine hétérogénéité des comportements subsiste, ce qui justifie leur étude dans la sous-section suivante.

Figure 2 : Evolution de la dispersion des contributions par catégories de périodes et par traitement ${ }^{5}$

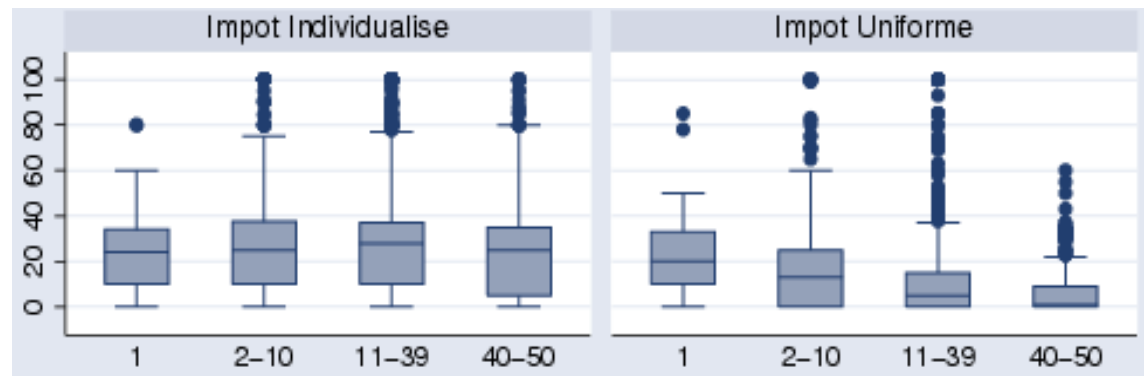

\subsection{Les déterminants de la coopération}

Afin d'analyser les déterminants des comportements individuels et leur dynamique, nous estimons pour chacun des deux traitements un modèle de type Tobit généralisé en panel avec erreurs aléatoires sur les périodes 2 à 50 (Tableau 2). Nous utilisons pour cela une procédure d'estimation en deux étapes. Une procédure d'estimation par maximum de vraisemblance à information complète en une étape serait certainement plus élégante, mais la procédure avec données de panel n'est pas triviale. L'estimateur en deux étapes nous permet de corriger un éventuel biais de sélection, en expliquant d'abord la décision de participer ou non à 
l'alimentation du fonds commun, puis, pour les seuls sujets qui ont fait le choix de participation, on estime les déterminants du montant contribué. Nous étudions les déterminants de contribuer un nombre non nul de jetons au fonds commun, à l'aide d'un modèle Probit en panel. Puis, conditionnellement à cette décision, nous expliquons le montant investi dans le fonds commun avec un modèle de Moindres Carrés Généralisés corrigé du biais de sélection ${ }^{6}$. Les variables exogènes propres à l'expérience incluent des variables instrumentales décalées d'une période, en particulier la contribution des autres membres du groupe et l'existence d'un sinistre. Une variable représente la différence entre le nombre effectif de dommages subis par un participant lors des périodes écoulées et le nombre théorique de dommages qu'il aurait dû subir, afin de mesurer l'influence de la (sous)sinistralité. Enfin, les variables de caractéristiques individuelles incluent l'âge, le sexe, le statut d'étudiant ou salarié, le niveau de diplôme, la spécialité disciplinaire en relation avec les mathématiques, la participation dans le passé à une expérience en économie, et la participation à une session organisée au Québec ou en France. Le contrôle pour le pays tient lieu d'effets de session ou de groupes. Le choix de la loterie est pris en compte comme indication de l'aversion au risque.

La probabilité de contribuer est fortement négativement liée à la période dans le traitement avec impôt uniforme (coefficient de -0,0154). Il en est de même dans le traitement individualisé, mais de façon négligeable (coefficient de -0,0067). La probabilité de contribuer est positivement liée à la contribution des autres dans le traitement avec impôt uniforme. Cette variable n'est pas statistiquement significative dans le traitement individualisé. Dans le traitement uniforme, la réciprocité est nécessaire pour inciter les participants à coopérer, dans la mesure où l'équilibre de Nash est une contribution nulle. Par ailleurs, puisque la fiscalité garantit la compensation des pertes, il est peu étonnant que la survenue d'un dommage à la période précédente n'ait pas d'effet significatif. En revanche, l'accumulation de dommages 
au-delà de la fréquence théorique a un effet négatif sur la probabilité de contribuer. Puisqu'il est de connaissance commune que les périodes sont indépendantes, ceci peut s'expliquer soit par une anomalie («l'illusion du parieur» qui pense que son risque va se réduire dans le futur), soit par une volonté de rattraper les pertes subies dans le passé en prenant davantage de risques.

Dans les régressions expliquant le montant des contributions conditionnellement à la volonté d'alimenter le fonds, le montant des contributions diminue avec la période du jeu pour l'impôt uniforme. Cette variable est sans effet dans le traitement de l'impôt individualisé. La contribution des autres à la période précédente n'a pas d'effet significatif dans le traitement uniforme. Pour le traitement individualisé, l'effet est négatif et significatif lorsque le fonds est déficitaire. Alors que dans le traitement uniforme le fonds est toujours déficitaire, la situation de déficit est observée dans $87 \%$ des cas dans le traitement avec impôt individualisé. Le coefficient de la variable croisée formée de la contribution des autres à la période précédente et une variable auxiliaire égale à 1 si le fonds était déficitaire, est significativement négatif. Ceci indique que si la contribution des autres est faible, on cherche à s'éviter une pénalité plus importante en contribuant davantage. A l'inverse, si les autres contribuent beaucoup, quand bien même le fonds s'est révélé déficitaire, les individus préfèrent resquiller. Cela ne leur permet toutefois de gagner légèrement plus que les autres que si le fonds n'est pas déficitaire lors de la période en cours. Une interprétation conforme à celle de Saijo et Nakamura (1995) requiert donc un fort optimisme de la part des resquilleurs. Une autre interprétation est que les joueurs maximisent en référence à des équilibres différents. 
Tableau 2. Probabilité et déterminants des contributions non nulles

\begin{tabular}{|c|c|c|c|c|c|c|c|c|}
\hline Traitements & \multicolumn{4}{|c|}{ Impôt Uniforme } & \multicolumn{4}{|c|}{ Impôt Individualisé } \\
\hline & \multicolumn{2}{|c|}{ Probit binaire en panel } & \multicolumn{2}{|c|}{ MCG en panel } & \multicolumn{2}{|c|}{ Probit binaire en panel } & \multicolumn{2}{|c|}{ MCG en panel } \\
\hline Variables & coeff & t-stat & coeff & t-stat & coeff & t-stat & coeff & t-stat \\
\hline Période & -0.0154 & -6.948 & -0.4147 & -7.579 & -0.0067 & -5.078 & 0.0118 & 0.372 \\
\hline Dommage en $\mathrm{t}-1$ & -0.0157 & -0.265 & 0.1551 & 0.255 & -0.1100 & -1.335 & 0.4073 & 0.537 \\
\hline $\begin{array}{l}\text { Dommages moins dommages } \\
\text { anticipés }\end{array}$ & -0.0318 & -1.805 & -0.3950 & -1.970 & -0.0301 & -2.757 & 0.0725 & 0.316 \\
\hline Contribution des autres en $\mathrm{t}-1$ & 0.1507 & 4.572 & 0.5880 & 1.058 & -0.0458 & -0.835 & -0.8958 & -1.566 \\
\hline $\begin{array}{l}\text { (Contribution des autres * } \\
\text { fonds déficitaire) en (t-1) }\end{array}$ & & & & & 0.0653 & 0.748 & -1.3788 & -1.828 \\
\hline Age & 0.0037 & 0.303 & 0.3377 & 1.492 & 0.0226 & 1.620 & -0.1740 & -0.603 \\
\hline Sexe (Réf. : hommes) & 0.3933 & 4.164 & 0.6501 & 0.249 & -0.8180 & -4.873 & 3.6670 & 0.928 \\
\hline Expérience & 0.9639 & 6.075 & 7.9556 & 1.631 & 0.0979 & 0.493 & 3.1537 & 1.035 \\
\hline Loterie & 0.0761 & 0.841 & 1.5924 & 0.697 & 0.1696 & 1.088 & 4.8156 & 1.624 \\
\hline $\begin{array}{l}\text { Étudiant (Réf. : salarié et } \\
\text { chômeur) }\end{array}$ & -0.8018 & -2.674 & -5.0881 & -1.208 & 0.6723 & 2.011 & 6.9353 & 1.159 \\
\hline Diplômé & -0.8854 & -5.171 & -6.0582 & -1.504 & 0.5690 & 2.745 & -5.5743 & -1.266 \\
\hline Formation en maths & 0.1088 & 1.011 & 2.2063 & 0.779 & 0.0846 & 0.318 & -0.6941 & -0.191 \\
\hline Québec (Réf. : France) & 0.2659 & 2.505 & 3.2854 & 1.377 & 0.2727 & 1.411 & 3.6309 & 1.142 \\
\hline Constante & 0.3934 & 0.857 & 4.8397 & 0.524 & 1.1976 & 1.954 & 21.6793 & 1.775 \\
\hline$\rho$ & 0.5747 & 19.180 & & & 0.5802 & 10.416 & & \\
\hline IRM & & & 12.2968 & 1.976 & & & -30.1040 & -0.980 \\
\hline $\mathrm{R}^{2}$ & & & \multicolumn{2}{|c|}{0.0930} & & & \multicolumn{2}{|c|}{0.0804} \\
\hline Observations & \multicolumn{2}{|c|}{3528} & \multicolumn{2}{|c|}{2187} & \multicolumn{2}{|c|}{3528} & \multicolumn{2}{|c|}{3030} \\
\hline $\begin{array}{l}\text { Valeur vraisemblance: } \\
\mathrm{V} \text {, contrainte } \\
\mathrm{V} \text {, du probit binaire }\end{array}$ & \multicolumn{2}{|c|}{$\begin{array}{l}-2342.99 \\
-2211.88 \\
-1599.59\end{array}$} & & & \multicolumn{2}{|c|}{$\begin{array}{c}-1436.10 \\
-1337.29 \\
-989.03\end{array}$} & & \\
\hline
\end{tabular}

Enfin, les variables démographiques n'exercent essentiellement aucune influence sur le montant contribué. En revanche, plusieurs variables ont un effet statistiquement significatif sur la probabilité de contribuer dans le traitement avec impôt uniforme. Cette probabilité augmente pour les hommes, avec la participation dans le passé à une expérience en économie, et la participation à une session organisée au Québec. Elle décroît assez fortement chez les sujets les plus diplômés (combinaison de la variable étudiant et diplômé). Cette situation est complètement inversée pour les hommes, et les sujets les plus diplômés dans le traitement avec impôt individualisé. Ces catégories de participants tendent davantage à se rapprocher de l'équilibre dans les deux traitements. 


\subsection{Des stratégies différenciées}

La significativité de l'effet individuel aléatoire et celle de certaines variables sociodémographiques laissent entrevoir une hétérogénéité des déterminants individuels de décision. Afin d'identifier les principales stratégies, une analyse par partitions (cluster analysis) a été entreprise. Celle-ci consiste à former des groupes homogènes distincts. Dans la première étape, trois variables ont été retenues pour établir le nombre de partitions : la fréquence des contributions non nulles, la moyenne et l'écart-type des contributions. En effet, il parait raisonnable de soutenir qu'un petit nombre de contributions non nulles, une moyenne et un écart-type des contributions faibles indiquent une stratégie de non coopération. Une fréquence élevée de contributions non nulles suggère au contraire une stratégie de coopération. L'écarttype des contributions identifie le caractère plus ou moins constant de la stratégie.

L'application de la méthode hiérarchique de Ward identifie 8 à 10 partitions résumant les stratégies des participants. Toutefois, deux partitions suffisent pour opposer des stratégies très différentes, sachant que les autres stratégies sont proches de la seconde partition ${ }^{7}$. Le Tableau 3 résume les statistiques caractérisant ces stratégies.

La partition 2 correspond à une stratégie de non coopération, caractérisée par un petit nombre de contributions non-nulles, une moyenne et un écart-type des contributions faibles. La partition 1 correspond à une stratégie coopérative, caractérisée par un nombre de contributions non nulles et une contribution moyenne nettement plus élevés. L'écart-type des contributions est grand, suggérant une stratégie hésitante, probablement liée aux réactions au comportement des membres du groupe. 
Tableau 3. Analyse des stratégies par partitions

\begin{tabular}{|c|c|c|c|c|c|}
\hline \multirow{2}{*}{$\begin{array}{l}\text { Traitements } \\
\text { Partitions }\end{array}$} & & \multicolumn{2}{|c|}{ Impôt Uniforme } & \multicolumn{2}{|c|}{ Impôt Individualisé } \\
\hline & & Partition 1 & Partition 2 & Partition 1 & Partition 2 \\
\hline \multicolumn{6}{|c|}{ Fréquence de contributions non nulles } \\
\hline & Moyenne & 42.33 & 15.67 & 48.74 & 38.54 \\
\hline & Écart-type & 6.57 & 8.05 & 2.00 & 11.81 \\
\hline \multicolumn{6}{|c|}{ Moyenne des contributions } \\
\hline & Moyenne & 14.52 & 4.89 & 40.16 & 17.34 \\
\hline & Écart-type & 9.29 & 3.40 & 10.30 & 8.40 \\
\hline \multicolumn{6}{|c|}{$\begin{array}{l}\text { Moyenne des écarts-type des } \\
\text { contributions }\end{array}$} \\
\hline & Moyenne & 12.20 & 8.56 & 17.56 & 14.25 \\
\hline & Écart-type & 7.24 & 5.60 & 8.02 & 7.15 \\
\hline Nombre d'observations & & 42 & 30 & 31 & 41 \\
\hline
\end{tabular}

Dans le traitement avec impôt individualisé, les caractéristiques de la partition 2 (stratégie non coopérative) sont assez proches de celles de la partition 1 du traitement avec impôt uniforme (stratégie coopérative). La seconde partition montre des participants avec un nombre élevé de contributions non nulles mais avec une moyenne de contribution à mi-chemin de l'optimum. En revanche, la partition 1 est caractérisée par une moyenne de contribution supérieure à l'optimum. En comparaison avec le traitement uniforme, la stratégie de coopération est plus hésitante. Dans les deux partitions, les écarts-types sont relativement plus élevés que ceux observés précédemment. Une analyse non paramétrique confirme, pour la partition 1, l'existence de différences entre le traitement avec impôt individualisé et l'autre traitement pour les trois variables retenues.

\section{Discussion et conclusion}

Dans cet article, nous avons représenté une situation économique impliquant des risques de dommage pour une partie de la population et la possibilité pour les agents de 
contribuer volontairement à l'alimentation d'un fonds permettant de compenser les victimes de ces dommages. Des exemples de telles situations sont nombreux. Ainsi en est-il dans le domaine de l'assurance-maladie où une bonne partie des cotisations perçues pour financer ces régimes est considérée par les contribuables comme acceptable (donc d'une certaine manière volontaire). Pourtant le déficit fréquent de ces régimes interroge sur leur mode de financement le plus adapté. Ces régimes de financement responsabilisent de manière très inégale les agents économiques à la prise en charge du coût de la protection sociale. Afin d'étudier l'efficacité comparée de plusieurs modes de financement des déficits de tels régimes, nous avons utilisé un jeu de bien public, dans lequel le déficit est absorbé soit par un impôt uniforme qui pénalise tous les agents de manière égalitaire, soit par un impôt individualisé en fonction des contributions volontaires des individus.

Notre protocole renforce le dilemme social par rapport au jeu standard de contribution volontaire au financement du bien collectif, puisque contribuer peut avoir ici un rendement marginal nul si l'on n'est pas victime d'un dommage. Si l'on conserve le parallèle avec l'assurance-maladie, il s'agit de cotiser alors même que l'on est en bonne santé. Bien que le dilemme social soit ainsi accru, l'évidence expérimentale tend à conforter la modélisation théorique et montre que la fiscalité individualisée réduit le risque de la coopération et responsabilise les individus. La fréquence des contributions nulles est considérablement réduite par rapport à une fiscalité homogène et le niveau moyen des contributions est très significativement supérieur. En effet, la fiscalité individualisée assure que la générosité des contributeurs sera moins exploitée par les autres. Certes, l'optimum n'est atteint en moyenne que dans à peine plus de $10 \%$ des périodes -rendant alors la taxation inutile-, mais les contributions moyennes s'en rapprochent. En revanche, la fiscalité uniforme, certes égalitaire puisqu'elle répartit à l'identique la charge des dommages entre tous les individus, 
déresponsabilise ces derniers et incite au comportement de passager clandestin, imposant de ce fait à la communauté un fardeau fiscal additionnel.

Il convient bien entendu de rester prudent quant à l'extrapolation de ces résultats. On pourrait ainsi soumettre le même protocole à une variation de la taille du groupe ou à une différenciation des dotations initiales. Tout en gardant à l'esprit ces limites, la comparaison entre nos deux traitements tend à soutenir les politiques recourant aux frais d'usager ou au ticket modérateur: l'individu participe individuellement au financement du programme d'aide selon son besoin du service. Tous les agents économiques ayant a priori la même probabilité de bénéficier du service, le financement par la fiscalité en assure l'existence alors que les frais d'usager viennent singulariser les utilisateurs effectifs. 


\section{Notes}

${ }^{1}$ Dans la version classique du jeu, l'équilibre de Nash correspond à une contribution nulle. Dès lors, des erreurs de jeu se confondent nécessairement avec une sur-contribution volontaire. Si l'équilibre est intérieur, les erreurs devraient se répartir également au dessus et en dessous de l'équilibre, s'annulant en moyenne.

${ }^{2} \mathrm{Si}$ le joueur maximise le profit actualisé sur $T$ périodes, le résultat du modèle ne change pas à cause de la séparabilité additive.

${ }^{3}$ Il existe en fait des équilibres de Nash non symétriques sans déficit budgétaire ex ante. Si un joueur croit que la contribution totale des N-1 autres joueurs serait déficitaire si lui-même ne contribue pour rien, ce joueur peut tout de même avoir intérêt dans certains cas à combler le déficit qu'il anticipe sur la contribution des autres. Pour les valeurs numériques des paramètres de l'expérience, les seuls déficits (en valeurs entières) qui correspondent à cette situation sont égaux à 1 et à 2 jetons, de sorte que la contribution nécessaire pour combler ce déficit serait presque nulle. De plus, de tels équilibres ne devraient pas être sélectionnés si les joueurs ont des anticipations rationnelles. Ils impliquent en effet de croire qu'un ou plusieurs autres joueurs aient "surcontribué" (plus que $\mathrm{L} / \mathrm{N}$ ), ce qui n'est pas une anticipation rationnelle dans notre expérience où les excédents budgétaires sont "brûlés".

${ }^{4}$ Puisque le surplus de contribution au delà du montant permettant de compenser toutes les victimes est brûlé, la peur du gaspillage et d'une perte d'efficacité sociale pourrait légitimement constituer une explication du faible niveau de contribution alternative à une interprétation en termes de resquillage. Cette interprétation est toutefois rejetée. En effet, l'apprentissage du jeu et la découverte de la situation quasi-permanente de déficit devraient conduire les individus à changer leur attitude s'ils étaient motivés par la crainte d'une perte d'efficience liée à la destruction du surplus.

${ }^{5}$ Les traits horizontaux de part et d'autre des barres représentent les valeurs adjacentes et les points représentent les observations isolées.

${ }^{6}$ L'aléa se décompose en un effet individuel aléatoire $\alpha_{i}$ et un aléa pur $\varepsilon_{i t}$ pour le probit expliquant les contributions non nulles. Nous retenons la variable période dans le probit comme variable explicative pour tenir compte de la dynamique des choix. Pour les régressions sur les contributions non nulles avec les Moindres Carrés Généralisés, une difficulté est reliée au caractère non cylindré des données dans cette deuxième étape. L'utilisation d'un terme aléatoire $\eta_{t}$ associé à l'effet période ajouté aux deux autres aléas a posé des difficultés de convergence. Nous avons retenu une spécification similaire au probit avec un effet individuel aléatoire et l'insertion de la variable période.

${ }^{7}$ Dans la mesure où nous voulons identifier les groupes de comportement les plus contrastés, nous avons utilisé un critère de différences comme moyen d'agrégation. La méthode du lien maximum (distance entre deux groupes donnée par celles entre les objets les plus éloignés dans les groupes) et celle de Ward (minimisation de la variance intra-groupe) sont les plus adaptées. Comme la méthode de Ward tend à produire de plus petits groupes, nous avons privilégié cette dernière, c'est-à-dire la méthode de minimisation de la somme au carré des erreurs (distance Euclidienne au carré $\left.\sum_{k=1}^{p}\left(x_{k i}-x_{k j}\right)^{2}\right)$. Concernant le choix du nombre de partitions, dans la mesure où nous voulons conserver les plus grandes différences entre 
groupes, nous examinons la courbe décroissante des distances entre partitions fusionnées à chaque étape. Cette courbe doit diminuer de façon monotone à mesure que des partitions proches sont fusionnées à chaque étape. Si lors d'une itération une différence nette se présente, alors il n'est pas raisonnable de grouper d'autres partitions. 


\section{Bibliographie}

Alm J. (1991). "On the Experimental Analysis of Taxpayer Reporting", The Accounting Review, vol. 66, $\mathrm{n}^{\circ} 3$, pp.577-593.

Andreoni J. (1993). "An Experimental Test of the Public Goods Crowding-out Hypothesis", American Economic Review, vol.83, n5, pp.1317-1327.

Bergstrom T. C., Blume, L. E. et Varian H. R. (1986). "On the Private Provision of Public Goods", Journal of Public Economics, vol.29, pp.25-49.

Chan K.S., Mestelman S., Moir R. et Muller R. A. (1999). "Heterogeneity and the Voluntary Provision of Public Goods", Experimental Economics, n³, pp.5-30.

Davis D. D. et Holt C. A. (1993). Experimental Economics. Princeton, Princeton University Press.

Goeree J. K., Holt C. A. et Laury S. K. (2000). "Private Costs and Public Benefits:Unraveling the Effects of Altruism and Noisy Behavior", Working Paper, University of Virginia, mimeo.

Holt C. A. et Laury S. K. (2000). "Theoretical Explanations of Treatment Effects in Voluntary Contributions Games", in C. R. Plott and V. Smith, Handbook of Experimental Economic Results. New York, Elsevier.

Isaac R.M. et Walker J. M. (1988). "Communication and Free-Riding Behavior: The Voluntary Contribution Mechanism", Economic Inquiry, n²6, pp.585-608.

Keser C. (1996). "Voluntary contributions to a public good when partial contribution is a dominant strategy", Economics Letters, n50, pp.359-366.

Ledyard J. O. (1995). "Public Goods: A Survey of Experimental Research", in J. H. Kagel and A. E. Roth, Handbook of Experimental Economics. Princeton, Princeton University Press.

Roberts R. D. (1987). "Financing Public Goods", Journal of Political Economy, vol.95, n², pp.420-437.

Saijo T. et Nakamura H. (1995). "The "Spite" Dilemma in Voluntary Contribution Mechanism Experiments", Journal of Conflict Resolution, vol.39, n³, pp.535-560.

Torgler B. (2004). "Speaking to Theorists and Searching for Facts: Tax Morale and Tax Compliance in Experiments", Working Paper, Université de Bâle.

Zeiliger R. (2000). "A presentation of Regate, Internet Based Software for Experimental Economics", http://www.gate.cnrs.fr/ zeiliger/regate/RegateIntro.ppt., GATE. 


\section{Annexe technique pour le traitement du déficit avec impôt uniforme}

Parmi les deux solutions possibles, seule une contribution nulle de la part de chacun des joueurs est un équilibre de Nash.

En effet, si $g_{j}=\frac{L}{N}, \forall j \neq i$, l'équation (1) s'écrit après simplification:

$\pi_{i}^{*}=100-\frac{L}{N^{2}}-\frac{N-1}{N} g_{i}-\beta-\frac{\alpha}{2 N}\left[\frac{L}{N}-g_{i}\right]^{2} \quad$ si $g_{i}<\frac{L}{N}$

S'il y a déficit budgétaire, le maximum de gain n'est sûrement pas atteint au voisinage de l'équilibre du budget puisqu'il vaut alors mieux éviter d'avoir à supporter un coût social en se plaçant tout de suite exactement à l'équilibre. Il se situe à une valeur plus basse, déterminée par calcul de la dérivée du profit (4) :

$$
\frac{\partial \pi_{i}}{\partial g_{i}}=-\left(\frac{N-1}{N}\right)+\frac{\alpha}{N}\left[\frac{L}{N}-g_{i}\right]
$$

Il en résulte que $\frac{\partial \pi_{i}}{\partial g_{i}}\left(g_{i}=0\right) \leq 0$, si : $\alpha L \leq N(N-1)$. Or, cette condition est forcément vérifiée si, comme nous l'avons supposé: $\alpha L \leq N-1$.

Pour que $g_{i}=0$ soit l'équilibre de Nash, il faut que le profit maximum atteint en ne contribuant pas au fonds commun dépasse le profit qui serait atteint en respectant l'équilibre budgétaire. En comparant ces deux valeurs du profit, on obtient la condition suivante :

$100-\frac{L}{N^{2}}-\beta-\frac{\alpha}{2 N} \frac{L^{2}}{N^{2}}>100-\frac{L}{N}$

ou plus simplement : $\beta<\frac{L}{N}\left[\frac{N-1}{N}-\frac{\alpha L}{2 N^{2}}\right]$

Si cette condition est respectée, $g_{i}=0$ définit l'équilibre unique du jeu. Or, elle est facilement respectée pour des valeurs raisonnables des paramètres $\alpha$ et $\beta$ et, en particulier, dans notre expérience. 


\section{Résumé court}

L'insuffisance de financement volontaire des biens collectifs pose la question de la responsabilité individuelle au sein des groupes et des sociétés. Une fiscalité renforçant l'engagement individuel permet-elle d'atténuer le problème du passager clandestin? Cet article compare expérimentalement l'efficacité de deux types de fiscalité visant à soutenir l'offre d'un bien collectif. Dans l'un, la charge fiscale est répartie égalitairement entre les sujets; dans l'autre, la charge est modulée en fonction du montant de la contribution volontaire individuelle. Les résultats montrent que l'uniformité incite au resquillage alors que l'individualisation fiscale favorise la coopération en responsabilisant les agents. Dans le second traitement, les contributions se rapprochent de l'optimum et la fréquence des déficits est réduite.

\section{Short abstract}

The financing deficit of collective goods questions the individual responsibility within groups and societies. Can a tax policy reinforcing individual responsibility alleviate the free riding behavior? By means of experimental data, this article compares the efficiency of two tax policies aiming at supporting the provision of collective goods. In one case, the tax burden is uniform for all the subjects; in the other case, the tax to be paid is modulated according to the voluntary contribution of the individual. The experimental results show that the uniform tax policy motivates free-riding more, whereas the individualized tax policy favors cooperation by increasing individual responsibility. In the latter case, individualized taxation brings the voluntary contributions and the optimum closer and reduces the frequency of deficits.

Codes JEL : H41, H21, H30, H50, C91.

Mots-clés: Bien collectif, fiscalité, responsabilité, expérimentation. 